\title{
Strong Edge Coloring of Cayley Graphs and Some Product Graphs
}

\author{
Suresh Dara ${ }^{1}$ - Suchismita Mishra ${ }^{2}$ Narayanan Narayanan ${ }^{3}$. \\ Zsolt Tuza ${ }^{4}$
}

Received: 19 October 2019/Revised: 17 July 2021 / Accepted: 9 August 2021 /

Published online: 2 February 2022

(C) The Author(s) 2022

\begin{abstract}
A strong edge coloring of a graph $G$ is a proper edge coloring of $G$ such that every color class is an induced matching. The minimum number of colors required is termed the strong chromatic index. In this paper we determine the exact value of the strong chromatic index of all unitary Cayley graphs. Our investigations reveal an underlying product structure from which the unitary Cayley graphs emerge. We then go on to give tight bounds for the strong chromatic index of the Cartesian product of two trees, including an exact formula for the product in the case of stars. Further, we give bounds for the strong chromatic index of the product of a tree with a cycle. For any tree, those bounds may differ from the actual value only by not more than a small additive constant (at most 2 for even cycles and at most 4 for odd cycles), moreover they yield the exact value when the length of the cycle is divisible by 4 .
\end{abstract}

Zsolt Tuza

tuza@dcs.uni-pannon.hu

Suresh Dara

suresh.dara@gmail.com

Suchismita Mishra

suchismitamishra6@gmail.com

Narayanan Narayanan

narayana@gmail.com

1 Department of Mathematics, School of Advanced Sciences, VIT Bhopal University,

Kothri Kalan, Sehore 466114, India

2 Computer Science and Engineering, Indian Institute of Technology Gandhinagar, Gujarat 382424, India

3 Department of Mathematics, Indian Institute of Technology Madras, Chennai 600036, India

4 Alfréd Rényi Institute of Mathematics, Budapest \& University of Pannonia, Veszprém, Hungary 
Keywords Edge coloring $\cdot$ Strong chromatic index $\cdot$ Cayley graph $\cdot$ Product graph

Mathematics Subject Classification $05 \mathrm{C} 15 \cdot 05 \mathrm{C} 76 \cdot 05 \mathrm{C} 05$

\section{Introduction}

Throughout the paper, an edge joining vertices $u$ and $v$ is denoted by $(u, v)$. Let $G$ be a simple, finite, undirected graph. A proper edge coloring is a map $c$ from the edge set of $G$ to a set of distinct colors such that for any two edges $(u, v)$ and $(v, w)$, $c((u, v)) \neq c((v, w))$. In addition, if $c((u, v)) \neq c((w, x))$ whenever $(v, w)$ is an edge, then $c$ is called a strong edge coloring. That is, a strong edge coloring is a proper edge coloring in which the vertex set of every color class induces a matching. The strong chromatic index of $G$, denoted by $\chi_{s}^{\prime}(G)$, is the minimum number of colors needed for any strong edge coloring of $G$.

There are several ways to look at this type of coloring. Namely, the following conditions are equivalent: (a) each color class is an induced matching, (b) each $P_{4} \subset G$ is 3-edge-colored, (c) the square $(L(G))^{2}$ of the line graph of $G$ is properly vertex-colored, (d) the coloring is 1-intersection edge coloring ${ }^{1}$ of $G$. The notion was introduced by Fouquet and Jolivet [18] with the intention to represent conflictfree channel assignment in some radio networks.

There are many interesting conjectures on this problem. Among them the most famous one is Erdős and Nešetríl's conjecture, which states that the strong chromatic index of any graph $G$ is at most $\left(5 \Delta^{2}-2 \Delta+1\right) / 4$ if $\Delta$ is odd, and at most $5 \Delta^{2} / 4$ if $\Delta$ is even. Here $\Delta=\Delta(G)$ denotes the maximum degree of the graph $G$. As mentioned in [16], the conjecture was raised at the end of 1985; the first publication proposing the bound $5 \Delta^{2} / 4$ seems to be [15]. If the bounds are valid, then they are tight, as shown by the graph obtained from the 5-cycle by substituting independent sets of size $\Delta / 2$ into its vertices if $\Delta$ is even (inserting a complete bipartite graph between any two consecutive sets along the cycle), or two consecutive sets of size $(\Delta+1) / 2$ and three others of size $(\Delta-1) / 2$ if $\Delta$ is odd.

Since there are well over a hundred papers dealing with the strong chromatic index, here we necessarily are limited to a partial survey only. The conjecture is proved by Chung et al. [12] for all $\Delta$ for the restricted class of graphs in which any two disjoint edges are joined by an edge (this particular case was conjectured already in 1983 by Bermond et al. [5], also raised independently in [15]).

For small $\Delta$, Andersen [3] and Horák et al. [20] showed that every cubic graph $G$ satisfies $\chi_{s}^{\prime}(G) \leq 10$. (The case of non-regular sub-cubic graphs is easy, as noted in [17]). The case of $\Delta=4$ is already complicated, the upper bound of 20 (respectively $19,18,17,16)$ is known to be valid only under the further assumption that the

\footnotetext{
${ }^{1}$ A $k$-intersection edge coloring, introduced in [33], is a proper edge coloring such that, for any two adjacent vertices, at most $k$ colors are incident with both.
} 
maximum average degree is at most 51/13 (respectively 15/4, 18/5, 7/2, 61/18) [30]. These are improvements of the estimates in [4], where e.g. the sufficiency of maximum average degree at most $19 / 5$ for 20-colorability was proved. The currently best result without average-degree restrictions states $\chi_{s}^{\prime}(G) \leq 21$, achieved only recently by Huang et al. [22]. Since each edge is strongly independent from all but at most 24 other edges, the upper bound 25 is very easy. Improving this bound to 24 , follows in one step from a general theorem of [12] quoted above, applying Brooks's theorem. This was further improved to 23 by Horák [19], to 22 by Cranston [13], and finally to 21 by Huang et al. [22].

Bruhn and Joos [9] proved that $\chi_{s}^{\prime}(G) \leq 1.93 \Delta^{2}(G)$, for graphs of sufficiently large maximum degree. This improves an old bound of $\chi_{s}^{\prime}(G) \leq 1.998 \Delta^{2}(G)$ proved by Molloy and Reed [32]. This bound is further improved for graphs with sufficiently large maximum degree. Hurley, de Joannis de Verclos and Kang [25] showed that the strong chromatic index is at most $1.772 \Delta^{2}(G)$, for any graph $\mathrm{G}$ with sufficiently large maximum degree $\Delta(G)$.

In 1989, Faudree et al. [16] conjectured that every bipartite graph $G$ satisfies $\chi_{S}^{\prime}(G) \leq(\Delta(G))^{2}$. Brualdi and Quinn Massey strengthened this conjecture to state that if $G$ is a bipartite graph with bipartition $(A, B)$ and $\Delta(A)$ and $\Delta(B)$ are the maximum degrees of the vertices in $A$ and $B$ respectively, then $\chi_{s}^{\prime}(G) \leq \Delta(A) \Delta(B)$ holds [8]. Later, Nakprasit [34] proved that this conjecture is valid when $\Delta(A)=2$. That is, for a $(2, \Delta)$-bipartite graph there is a strong edge coloring that uses at most $2 \Delta$ colors. In 2017 , Huang et al. [23] showed that if $G$ is a $(3, \Delta)$-bipartite graph, then $\chi_{s}^{\prime}(G) \leq 3 \Delta$. Bipartite graphs are complex also in the algorithmic sense: Mahdian [31] showed that determining the exact value of the strong chromatic index is NP-hard even for bipartite graphs with girth at least $g$, for any natural number $g$.

It is known that every planar graph admits a $4 \Delta+4$ strong edge coloring [17]. Moreover, $3 \Delta+5$ colors are sufficient if the planar graph has girth 6 , and if it has girth at least 7 then even $3 \Delta$ colors are sufficient [24]. Furthermore for outerplanar graphs an exact formula can be given, as shown in the following theorem.

Theorem 1 [7] Let $G$ be an outerplanar graph. Then $\chi_{s}^{\prime}(G)=\max \left\{\max _{u v \in E}\right.$ $\left.d(u)+d(v)-1, \max _{H \in \mathcal{P}} \chi_{s}^{\prime}(H)\right\}$, where $\mathcal{P}$ is the set of all puffer subgraphs of $G$. Moreover, if $G$ is bipartite, then $\chi_{s}^{\prime}(G)$ is either $\max _{u v \in E} d(u)+d(v)-1$ or $\max _{u v \in E} d(u)+d(v)$.

The exact values for the puffer graphs are obtained in [11].

A Halin graph is a plane graph constructed from a tree $T$ without vertices of degree two by connecting all leaves through a cycle $C$. Let $G=T \cup C$ be a Halin graph. Lai et al. [28] proved that $\chi_{s}^{\prime}(G) \leq \chi_{s}^{\prime}(T)+3$, provided $G$ is different from some special graphs. Also it is known that every Halin graph $G$ with $\Delta(G) \geq 4$ satisfies $\chi_{s}^{\prime}(G) \leq 2 \Delta(G)+1$ [21]. Moreover, apart from two exceptions, cubic Halin graphs have $\chi_{s}^{\prime}(G) \leq 7$ [29].

We first discuss the strong edge coloring of the class of graphs called unitary Cayley graph. Let $U_{n}$ be the set of all units of $\mathbb{Z}_{n}$, that is $U_{n}=\left\{m \in \mathbb{Z}_{n} \mid \operatorname{gcd}(m, n)=1\right\}$. For any natural number the Cayley graph 
$\operatorname{Cay}\left(\mathbb{Z}_{n}, U_{n}\right)$ is called a unitary Cayley graph and is denoted by $X_{n}$. Now for any $m \in \mathbb{Z}_{n}, \operatorname{gcd}(m, n)=1$ if and only if $\operatorname{gcd}(-m, n)=1$, and every element of $U_{n}$ generates $\mathbb{Z}_{n}$. Hence, $X_{n}$ is an undirected connected (and also Hamiltonian) graph.

The structure and various properties of unitary Cayley graphs have been studied in the literature (see $[6,27]$ ). For a natural number $n, X_{n}$ is a $\phi(n)$-regular graph, where $\phi$ is Euler's phi function. It is known that a unitary Cayley graph is bipartite if and only if $n$ is even [14]. Akhtar et al. [1] showed that the chromatic index of $X_{n}$ is $\phi(n)+1$ if $n$ is odd, and it is $\phi(n)$ otherwise.

Here we determine exactly the strong chromatic index of Cayley graphs $X_{n}$, for all $n$. Namely, we prove that if a given natural number $n$ has $k$ distinct prime factors in its prime factor decomposition, then $\chi_{s}^{\prime}\left(X_{n}\right)=|E| / 2^{k-1}$.

Besides the unitary Cayley graphs we also discuss about the Cartesian product of graphs. Cartesian product of graphs is an important notion in the theory of graph products, where the structure of the factors (graphs) appears as an induced subgraph. For several important results and properties of the Cartesian product see the book [26]. Here we concentrate on the Cartesian product of certain classes of graphs. The first result in the literature concerning strong chromatic index under the product operation was given in [17] where the exact value for the $n$-dimensional hypercube was proven to be $\chi_{s}^{\prime}\left(Q_{n}\right)=2 n$ for all $n \geq 2$. The systematic study of $\chi_{s}^{\prime}$ on various types of graph products was initiated by Togni in [35]; in particular, he determined formulas for the Cartesian products of paths and cycles. Complexity issues and general inequalities for some product types have also been presented by Chalermsook et al. in [10].

Here we give both upper and lower bounds for the strong chromatic index of the Cartesian product of any two trees, and for the product of any tree with any cycle. If the length of a cycle $C$ is a multiple of 4 then our results give the exact value of the strong chromatic index of the product of $C$ with an arbitrary tree.

\section{Definitions and Notation}

Following the standard notation, $C_{\ell}$ denotes the cycle graph of length $\ell$. We write $u \sim v$ to say $u$ is adjacent to $v$.

For any edge $(u, v)$ in a graph $G$, define the edge degree $d^{\prime}(u, v)$ of $(u, v)$ to be the number of edges incident either to $u$ or to $v$. That is, $d^{\prime}(u, v)=d(u)+d(v)-1$. The maximum edge degree of a graph, denoted by $\Delta^{\prime}(G)$, is the maximum of all the edge degrees.

Recall that the Cartesian product of any two graphs $G$ and $H$, denoted by $G \square H$, is the graph with vertex set $\{a: u \mid a \in V(G)$ and $u \in V(H)\}$, where two vertices $a: u$ and $b: v$ are adjacent if either $a=b$ and $u$ is adjacent to $v$ in $H$, or $u=v$ and $a$ is adjacent to $b$ in $G$.

For any vertex $h$ of $H$ define a $G$-fiber $G \times\{h\}$ to be the graph with vertex set $\{a: h \mid a \in V(G)\}$ and edge set $\{(a: h, b: h) \mid(a, b) \in E(G)\}$. Similarly define $H$ fibers. Note that we can write $G \square H$ as $(G \times V(H)) \cup(V(G) \times H)$.

Another type of graph product is the categorical product or direct product of $G$ and $H$, denoted by $G \times H$. It also has the vertex set $\{a: u \mid a \in V(G)$ and 
$u \in V(H)\}$, but in this product vertices $a: u$ and $b: v$ are adjacent if $a \sim b$ in $G$ and $u \sim v$ in $H$. Notation is expressive for both kinds of products, indicating that $K_{2} \square K_{2} \cong C_{4}$ and $K_{2} \times K_{2} \cong 2 K_{2}$.

\section{Strong Edge Coloring of Unitary Cayley Graphs}

In this section we determine the strong chromatic index of all unitary Cayley graphs.

From its definition, it follows that the Cayley graph $X_{n}$ is a $\phi(n)$-regular graph of order $n$. Therefore, the size (number of edges) of $X_{n}$ is $n \frac{\phi(n)}{2}$. Note that if $n$ has prime divisors $p_{1}, \ldots, p_{k}$ then $\phi(n)=n \prod_{i=1}^{k}\left(1-\frac{1}{p_{i}}\right)$. It will also be convenient to introduce the notation $n^{\prime}=\frac{n}{\prod_{i=1}^{k} p_{i}}$, and to write $n^{\prime \prime}=\prod_{i=1}^{k} p_{i}$.

Our main result is the following formula.

Theorem 2 Let $n$ be a natural number with the prime factorization $p_{1}^{r_{1}} p_{2}^{r_{2}} \ldots p_{k}^{r_{k}}$. Then $\chi_{s}^{\prime}\left(X_{n}\right)=\frac{|E|}{2^{k-1}}=n \frac{\phi(n)}{2^{k}}$.

Proof We prove that $\frac{|E|}{2^{k-1}}$ is both an upper bound and a lower bound on $\chi_{s}^{\prime}\left(X_{n}\right)$. First let us make some observations on the structure of $X_{n}$. Each $v \in \mathbb{Z}_{n}$ can be classified according to its residues modulo the prime factors, assigning with $v$ the $k$-tuple

$$
\langle v\rangle:=\left(\bmod p_{1}, \bmod p_{2}, \ldots, \bmod p_{k}\right) .
$$

This assignment partitions the vertex set into $n^{\prime \prime}=p_{1} p_{2} \ldots p_{k}$ classes, each class having cardinality $n^{\prime}=p_{1}^{r_{1}-1} p_{2}^{r_{2}-1} \ldots p_{k}^{r_{k}-1}$. These are precisely the classes of false twins: if $\langle u\rangle=\langle v\rangle$, then $u$ and $v$ are not adjacent but they have exactly the same neighborhood.

As it can be seen directly from the definition, non-adjacency means that the two numbers in question are incongruent modulo each $p_{i}$. In this way we can represent $X_{n}$ with a $k$-dimensional box, first taking the categorical product graph $K^{*}:=K_{p_{1}} \times K_{p_{2}} \times \cdots \times K_{p_{k}}$, and then substituting independent sets of size $n^{\prime}$ (sets of false twins) into the product graph; that is, each edge of $K^{*}$ is enlarged to an induced copy of the complete bipartite graph $K_{n^{\prime}, n^{\prime}}$. (This graph only rarely happens to be a product graph after substitution.)

Let us note further that if $n=p^{r}$, i.e. in case $n$ has just one prime divisor, $X_{n}$ is the complete $p$-partite graph in which each vertex class has $p^{r-1}$ vertices. Then no two edges of $X_{n}$ can form an induced matching, and the unique strong edge coloring assigns a distinct color to each edge. For this reason,

$$
\chi_{s}^{\prime}\left(X_{p^{r}}\right)=\left|E\left(X_{p^{r}}\right)\right|=\frac{p^{2 r-1}(p-1)}{2}
$$

clearly holds, and we only have to consider $k \geq 2$.

We first prove the upper bound given in the theorem.

Proof of the upper bound:

We show that the edge set of $X_{n}$ can be partitioned into induced matchings of size 
$2^{k-1}$. This clearly implies the inequality $\chi_{s}^{\prime} \leq \frac{|E|}{2^{k-1}}$.

To this end, we construct an edge partition for $K^{*}$, where the graph $K^{*}$ is actually just $X_{n^{\prime \prime}}$. Consider any edge $(u, v) \in K_{p_{1}} \times \cdots \times K_{p_{k}}$. Let us write $\langle u\rangle$ and $\langle v\rangle$ in the form $\langle u\rangle=\left(a_{1}, \ldots, a_{k}\right)$ and $\langle v\rangle=\left(b_{1}, \ldots, b_{k}\right)$. Since $(u, v)$ is an edge, we have $a_{i} \neq b_{i}$ for all $1 \leq i \leq k$. Hence, the Cartesian product $\left\{a_{1}, b_{1}\right\} \times \cdots \times\left\{a_{k}, b_{k}\right\}$ specifies a set, say $S$, of exactly $2^{k}$ vertices in $K^{*}$. We claim that the subgraph induced by $S$ is a matching of size $2^{k-1}$. Indeed, two vertices $\left(a_{1}^{\prime}, \ldots, a_{k}^{\prime}\right),\left(b_{1}^{\prime}, \ldots, b_{k}^{\prime}\right) \in S$ are adjacent if and only if $\left\{a_{i}^{\prime}, b_{i}^{\prime}\right\}=\left\{a_{i}, b_{i}\right\}$ holds for all $i$, as otherwise the corresponding two numbers would share a prime divisor. Hence every $v \in S$ has one and only one neighbor in $S$; that is, $S$ induces a matching, which then necessarily has $|S| / 2=2^{k-1}$ edges. Note further that every edge induced by $S$ determines exactly the same set $S$, and so each edge of $K^{*}$ belongs to precisely one induced matching defined in this way.

Substituting independent sets of size $n^{\prime}$ into the vertices of $K^{*}$, each edge gets replaced by a subgraph isomorphic to $K_{n^{\prime}, n^{\prime}}$. One such example is shown in Fig. 1. Therefore each induced matching of size $2^{k-1}$ from $K^{*}$ becomes an induced subgraph isomorphic to $2^{k-1} K_{n^{\prime}, n^{\prime}}$ in $X_{n}$. Since the induced matchings defined in $K^{*}$ are mutually edge-disjoint, these copies of $2^{k-1} K_{n^{\prime}, n^{\prime}}$ are edge-disjoint. Obviously each of these subgraphs can be decomposed into $\left(n^{\prime}\right)^{2}$ edge-disjoint induced matchings of size $2^{k-1}$. Consequently we obtain a required edge partition of $X_{n}$, and the upper bound $\chi_{s}^{\prime} \leq \frac{|E|}{2^{k-1}}$ follows.

In the proof of the lower bound we shall apply a particular case of the following theorem due to Alon [2]. Originally the result was stated for pairs of k-tuples of sets, we rewrite it with a somewhat simpler formalism dealing with pairs of sets.
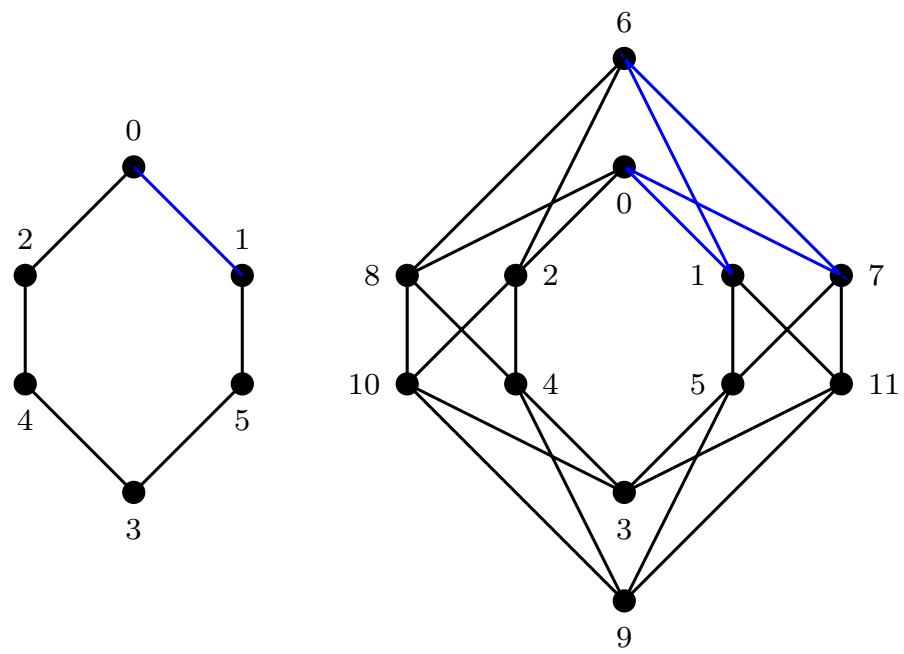

Fig. 1 Substitution of an edge of $X_{6}$ in $X_{12}$ 
Lemma 1 [2] Let $Y_{1}, \ldots, Y_{k}$ be mutually disjoint sets, $Y=Y_{1} \cup \cdots \cup Y_{k}$. Moreover let $s_{1}, \ldots, s_{k}$ and $t_{1}, \ldots, t_{k}$ be positive integers. Assume that $\left(A_{1}, B_{1}\right), \ldots,\left(A_{m}, B_{m}\right)$ are pairs of sets with the following properties:

- $A_{i} \cup B_{i} \subseteq Y$ and $A_{i} \cap B_{i}=\emptyset$ for all $1 \leq i \leq m$;

- $A_{i} \cap B_{j} \neq \emptyset$ for all $1 \leq i<j \leq m$;

- $\left|A_{i} \cap Y_{\ell}\right| \leq s_{\ell}$ and $\left|B_{i} \cap Y_{\ell}\right| \leq t_{\ell}$ for all $1 \leq i \leq m$ and all $1 \leq \ell \leq k$.

Then $m \leq \prod_{\ell=1}^{k}\left(\begin{array}{c}s_{\ell}+t_{\ell} \\ s_{\ell}\end{array}\right)$

Note that in case of $s_{1}=\ldots=s_{k}=t_{1}=\ldots=t_{k}=1$ the upper bound simply means $m \leq 2^{k}$.

\section{Proof of the lower bound:}

We prove that the largest induced matchings in $X_{n}$ have no more than $2^{k-1}$ edges. This clearly imply the lower bound $\chi_{s}^{\prime} \geq \frac{|E|}{2^{k-1}}$.

Assume that the edges $\left(u_{1}, v_{1}\right), \ldots,\left(u_{h}, v_{h}\right)$ form an induced matching. We further define $2 h$ pairs of sets $\left(A_{i}, B_{i}\right)$ over an underlying set $Y=Y_{1} \cup \cdots \cup Y_{k}$; two pairs of sets are to be defined for each edge. For every $1 \leq \ell \leq k$ we write $Y_{\ell}=\left\{y_{0}^{\ell}, y_{1}^{\ell}, \ldots, y_{p_{\ell-1}}^{\ell}\right\}$; those vertices represent the residue classes modulo $p_{\ell}$.

The ordered $k$-tuple $\langle v\rangle$ of integers allows us to associate a $k$-element subset $H(v) \subset Y$ with each vertex $v \in X_{n}$ as follows:

$$
H(v)=\left\{y_{\bmod p_{1}}^{1}, y_{\bmod p_{2}}^{2}, \ldots, y_{\bmod p_{k}}^{k}\right\} .
$$

Now, for every $1 \leq i \leq h$ we set

$$
A_{2 i-1}=H\left(u_{i}\right), \quad B_{2 i-1}=H\left(v_{i}\right), \quad A_{2 i}=H\left(v_{i}\right), \quad B_{2 i}=H\left(u_{i}\right) .
$$

Then the sets of the same index are disjoint, due to the adjacency of $u_{i}$ and $v_{i}$. On the other hand, since $u_{i}$ is not adjacent to $u_{j}$ if $j \neq i$, the difference $u_{i}-u_{j}$ is divisible by some $p_{\ell}$, which implies that $A_{2 i-1}$ - as well as $B_{2 i}$ - meets both $B_{2 j-1}$ and $A_{2 j}$ inside $Y_{\ell}$. Analogous consequences can be derived from the nonadjacencies $u_{i} / \sim v_{j}$, $v_{i} / \sim u_{j}$, and $v_{i} / \sim v_{j}$. It follows that the pairs $\left(A_{1}, B_{1}\right), \ldots,\left(A_{2 h}, B_{2 h}\right)$ satisfy the conditions of Lemma 1 , with $s_{\ell}=t_{\ell}=1$ for all $1 \leq \ell \leq k$. Consequently $2 h \leq 2^{k}$ holds, so that every induced matching has at most $2^{k-1}$ edges, as claimed. This completes the proof of the theorem.

\section{Cartesian Product of Two Trees}

In this section, we give both a lower and an upper bound for the strong chromatic index of the Cartesian product of two trees. First we prove that twice the maximum degree is an upper bound for the strong chromatic index of the product of two trees. Recall Brualdi and Quinn Massey's conjecture [8] for bipartite graphs, namely that 
the strong chromatic index of an $(A, B)$-partite graph is at most $\Delta(A) \Delta(B)$. Note that the product of two trees is a bipartite graph. So our result implies that the product of two trees satisfies the conjectured bound given by Brualdi and Quinn Massey.

Theorem 3 Let $T_{1}$ and $T_{2}$ be two trees. Then $\chi_{s}^{\prime}\left(T_{1} \square T_{2}\right) \leq 2 \Delta\left(T_{1} \square T_{2}\right)$.

Proof Let $x$ and $y$ be leaves of $T_{1}$ and $T_{2}$ respectively. We consider $T_{1}$ and $T_{2}$ as rooted trees with roots $x$ and $y$ respectively. Each of $x$ and $y$ has unique neighbors in their respective trees. Let $a$ be the only neighbor of $x$ in $T_{1}$ and $c$ be that of $y$ in $T_{2}$.

We know that $2 \Delta\left(T_{1} \square T_{2}\right)=2 \Delta\left(T_{1}\right)+2 \Delta\left(T_{2}\right)$. Now onwards we write $\Delta_{1}$ for $\Delta\left(T_{1}\right)$ and $\Delta_{2}$ for $\Delta\left(T_{2}\right)$. We give a coloring to the edges of $T_{1} \square T_{2}$. The Cartesian product of the subgraph $S_{1}$, induced by $\{x, a\}$ in $T_{1}$ and the subgraph $S_{2}$, induced by $\{y, c\}$ in $T_{2}$ is just a 4-cycle. Now give colors $2 \Delta_{1}-1,2 \Delta_{1}, 2 \Delta_{1}+2 \Delta_{2}-1,2 \Delta_{1}+$ $2 \Delta_{2}$ to $(x: y, a: y),(a: c, x: c),(x: c, x: y),(a: y, a: c)$ respectively.

The idea is to construct a tower of induced subgraphs $G_{1}=S_{1} \square S_{2} \subseteq G_{2} \subseteq$ $G_{3} \subseteq \ldots \subseteq G_{n}=T_{1} \square T_{2}$ of $T_{1} \square T_{2}$ and in each step we extend the coloring of $G_{i-1}$ to a strong edge coloring of $G_{i}$, so that the coloring satisfies the following conditions:

(i) All the edges of $T_{1} \times V\left(T_{2}\right)$ (recall it is collection of all the edges of the $T_{1}$ fibers) use colors from $\left\{1,2,3, \ldots, 2 \Delta_{1}\right\}$.

(ii) All the edges of $V\left(T_{1}\right) \times T_{2}$ use colors from $\left\{2 \Delta_{1}+1,2 \Delta_{1}+2, \ldots, 2 \Delta_{1}+2 \Delta_{2}\right\}$.

(iii) The distance from $v$ to $v^{\prime}$ is even in $T_{2}$ if and only if for every $\left(u, u^{\prime}\right) \in E\left(T_{1}\right)$, the color of the edge $\left(u: v, u^{\prime}: v\right)$ is same as the color of $\left(u: v^{\prime}, u^{\prime}: v^{\prime}\right)$.

(iv) The distance from $u$ to $u^{\prime}$ is even in $T_{1}$ if and only if for every $\left(v, v^{\prime}\right) \in E\left(T_{2}\right)$, the color of the edge $\left(u: v, u: v^{\prime}\right)$ is same as the color of $\left(u^{\prime}: v, u^{\prime}: v^{\prime}\right)$.

Loosely speaking this will be carried out by visiting carefully selected representative vertices in a sequence where the incident uncolored edges are in an interaction with relatively few previously colored edges; and then copy the colors to further subsets of edges in the current subgraph. The procedure will be performed on $T_{1}$ and $T_{2}$ separately. This approach can be formalised in a precise way as described below.

We define $G_{2}$ to be the subgraph induced by $\left\{u: v \mid d_{T_{1}}(x, u) \leq 2, d_{T_{2}}(y, v) \leq 2\right\}$ of $T_{1} \square T_{2}$. Now $\mid\left\{\left(a: y, a^{\prime}: y\right),\left(a: c, a^{\prime}: c\right) \mid a^{\prime} \quad\right.$ is adjacent to $a$ and $\left.a^{\prime} \neq x\right\} \mid \leq 2 \Delta_{1}-2$. So we can assign colors $1,2, \ldots, 2 \Delta_{1}-2$ to these edges such that no two edges get the same color. Similarly we can distribute the colors $2 \Delta_{1}+$ $1,2 \Delta_{1}+2, \ldots, 2 \Delta_{1}+2 \Delta_{2}-2$ to $\left\{\left(x: c, x: c^{\prime}\right),\left(a: c, a: c^{\prime}\right) \mid c^{\prime}\right.$ is adjacent to $c$ and $\left.c^{\prime} \neq y\right\}$, so that no two edges get the same color. 


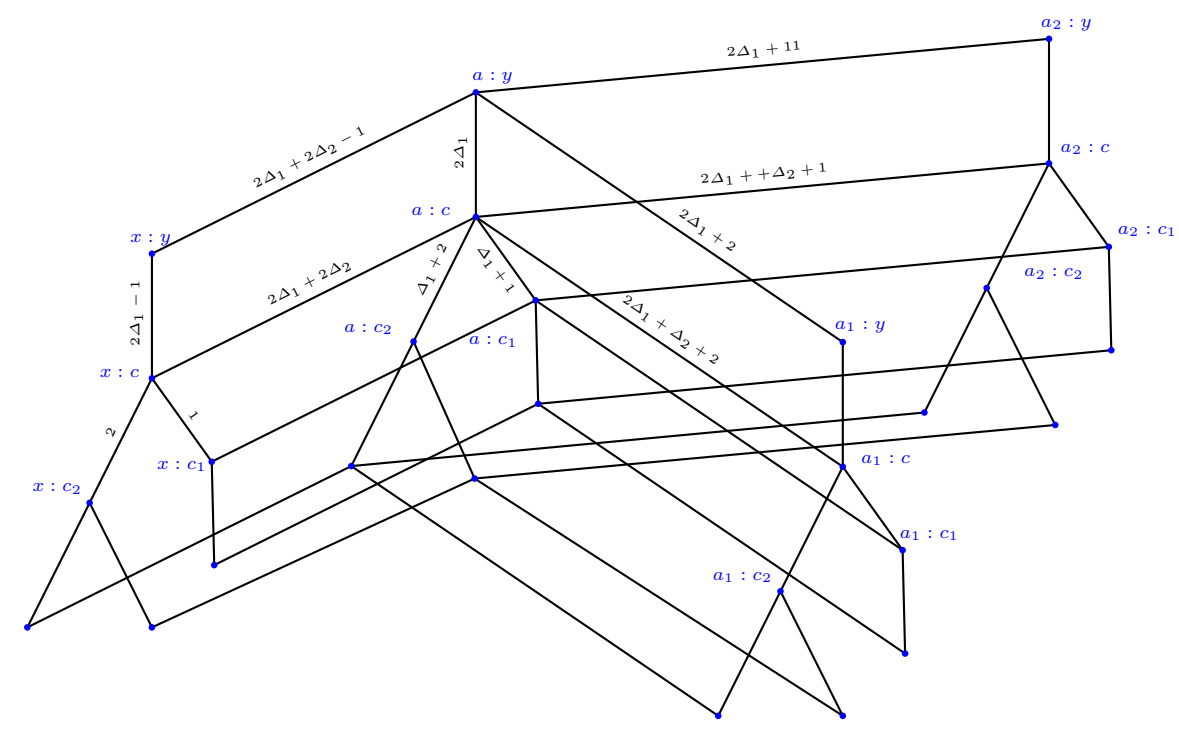

Since $T_{1}$ is a tree, if $a^{\prime}$ and $a^{\prime \prime}$ are adjacent to $a$, then they are not adjacent to each other, therefore $\left\{\left(a^{\prime}: y, a^{\prime}: c\right) \mid a^{\prime} \sim a\right\}$ forms an induced matching; and so does $\left\{\left(a^{\prime}: c, a^{\prime}: c^{\prime}\right) \mid a^{\prime} \sim a\right\}$ for any fixed neighbor $c^{\prime}$ of $c$ in $T_{2}$. Hence we can assign the color of $(x: y, x: c)$ to $\left\{\left(a^{\prime}: y, a^{\prime}: c\right) \mid a^{\prime} \neq x, a^{\prime} \sim a\right\}$ and the color of $(x$ : $\left.c, x: c^{\prime}\right)$ to $\left\{\left(a^{\prime}: c, a^{\prime}: c^{\prime}\right) \mid a^{\prime} \neq x, a^{\prime} \sim a\right\}$. Similarly, for every fixed $a^{\prime} \sim a$, we can give the color of $\left(a^{\prime}: y, a: y\right)$ to $\left\{\left(a: c^{\prime}, a^{\prime}: c^{\prime}\right) \mid c^{\prime} \neq y, c^{\prime} \sim c\right\}$. Clearly this coloring satisfies (i), (ii), (iii), (iv).

Now for every $\ell$, define $G_{\ell}$ to be the subgraph induced by $\{u: v \mid$ $\left.d_{T_{1}}(x, u) \leq \ell, d_{T_{2}}(y, v) \leq \ell\right\} \quad$ (note that if $m=\max \left\{\operatorname{diam}\left(T_{1}\right), \operatorname{diam}\left(T_{2}\right)\right\}$, then $G_{m}=T_{1} \square T_{2}$ ). Suppose we have a coloring of $G_{\ell-1}$ that satisfies (i), (ii), (iii), (iv). Now we give coloring to the rest of the edges of $G_{\ell}$. First we assign the colors to $\left\{\left(u: v, u^{\prime}: v\right) \mid\left(u, u^{\prime}\right) \in E\left(T_{1}\right), v \in V\left(T_{2}\right)\right\}$.

Let $u$ be a vertex of $T_{1}$ of distance $\ell-1$ from $x$ and $s$ be its parent. The colored edges of distance two from $\left\{\left(u: y, u^{\prime}: y\right) \mid u^{\prime} \neq s, u^{\prime} \sim u\right\}$ are the edges incident to $s: y$ or $u: c$. Since $G_{\ell-1}$ satisfies (i) and (ii), so $(s: c, u: c)$ is the only edge adjacent to $u$ : $c$ that uses a color among $1,2,3, \ldots, 2 \Delta_{1}$. Again, coloring of $G_{i-1}$ satisfies (iii), there are at least $\Delta_{1}-1$ colors among $\left\{1,2,3, \ldots, 2 \Delta_{1}\right\}$, not being used by any of the edges incident to $s: y$ or by the edge $(s: c, u: c)$. We can assign those colors to $\left\{\left(u: y, u^{\prime}: y\right) \mid u^{\prime} \neq s, u^{\prime} \sim u\right\}$, so that no two edges get the same color. Then for any fixed $u^{\prime} \neq s$ where $u^{\prime} \sim u$, the only colored edges of distance two from $\left(u: c, u^{\prime}: c\right)$ are the edges incident to $s: c$ or incident to one of the vertices of the set $\left\{u: c^{\prime} \mid c^{\prime} \sim c\right\}$. Now for any $c^{\prime} \neq y$ adjacent to $c,\left(s: c^{\prime}, u: c^{\prime}\right)$ is the only edge incident to $u: c^{\prime}$ that uses colors among $1,2,3, \ldots, 2 \Delta_{1}$ (by (i), (ii)). Again (iii) says that the color of $\left(s: c^{\prime}, u: c^{\prime}\right)$ is the color of $(s: y, u: y)$. Therefore, there are $\Delta_{1}-1$ suitable colors among $1,2,3, \ldots, 2 \Delta_{1}$ for $\left\{\left(u: c, u^{\prime}: c\right) \mid u^{\prime} \neq s, u^{\prime} \sim u\right\}$. Distribute those colors to 
$\left\{\left(u: c, u^{\prime}: c\right) \mid u^{\prime} \neq s, u^{\prime} \sim u\right\}$.

Now we claim the following for a fixed $u^{\prime} \neq s$, where $u^{\prime}$ is adjacent to $u$.

Claim We can assign the color of $\left(u: v, u^{\prime}: v\right)$ to $\left\{\left(u: t, u^{\prime}: t\right) \mid d_{T_{2}}(v, t)=2\right\}$.

Proof of the Claim We apply induction on distance from $u$. If $\left(u: t, u^{\prime}: t\right)$ is already colored, then the induction hypothesis assures the claim. Now we may assume that $\left(u: t, u^{\prime}: t\right)$ did not get a color till now and $v^{\prime}$ is adjacent to both $v$ and $t$. The only colored edges of distance two from $\left(u: t, u^{\prime}: t\right)$ are $\left\{\left(s: t^{\prime}, u: t^{\prime}\right) \mid t^{\prime}\right.$ is a child of $\left.t\right\}$ and the edges incident to $u: v^{\prime}$ or $s: t$. Since the coloring of $G_{\ell-1}$ satisfies (iii), the only colors which may create problems are the colors of the edges incident to $u: v^{\prime}$ or $s: t$.

Since $v^{\prime}$ is adjacent to $t$, the color of $\left(u: v, u^{\prime}: v\right)$ is not used by any of the edges incident to $u: v^{\prime}$. Also (iii) ensures that it is not used by the edges incident to $s: t$. Again $\left\{\left(u: t, u^{\prime}: t\right) \mid d_{T_{2}}(v, t)=2\right\}$ is an induced matching. Hence we can assign the color of $\left(u: v, u^{\prime}: v\right)$ to $\left\{\left(u: t, u^{\prime}: t\right) \mid d_{T_{2}}(v, t)=2\right\}$. The claim is proved. By coloring accordingly we get a partial strong edge coloring that satisfies (i) and (iii).

By symmetry we can color $V\left(T_{1}\right) \times T_{2}$ by using the colors $2 \Delta_{1}+1,2 \Delta_{1}+$ $2, \ldots, 2 \Delta_{1}+2 \Delta_{2}$ such that it satisfies (ii) and (iv). That says the strong chromatic index of $G_{n}=T_{1} \square T_{2}$ is at most $2 \Delta_{1}+2 \Delta_{2}=2 \Delta\left(T_{1} \square T_{2}\right)$.

In the above theorem we observed an upper bound for the Cartesian product of two trees. This bound is tight. That is there exist trees $T_{1}$ and $T_{2}$ such that $\chi_{s}^{\prime}\left(T_{1} \square T_{2}\right)=2 \Delta\left(T_{1} \square T_{2}\right)$. Before showing that, we find a lower bound for the same, in the next theorem.

Theorem 4 Let $T_{1}$ and $T_{2}$ be two trees. Then

$$
\chi_{s}^{\prime}\left(T_{1} \square T_{2}\right) \geq \max \left\{2 \Delta\left(T_{1}\right)+\Delta^{\prime}\left(T_{2}\right), 2 \Delta\left(T_{2}\right)+\Delta^{\prime}\left(T_{1}\right)\right\}+1=\Delta^{\prime}\left(T_{1} \square T_{2}\right)+1 .
$$

Proof Let $(a, b)$ be an edge with maximum edge degree in $T_{1}$. Also let $c$ be a vertex of maximum degree in $T_{2}$ and $d$ be a neighbor of it. Now the edge $(a: d, b: d)$ and all the edges incident to $a: c$ or $b: c$ should get different colors. So $\chi_{s}^{\prime}\left(T_{1} \square T_{2}\right) \geq \Delta^{\prime}\left(T_{1}\right)+2 \Delta\left(T_{2}\right)+1$. Similarly, $\chi_{s}^{\prime}\left(T_{1} \square T_{2}\right) \geq \Delta^{\prime}\left(T_{2}\right)+2 \Delta\left(T_{1}\right)+1$. Therefore,

$\chi_{s}^{\prime}\left(T_{1} \square T_{2}\right) \geq \max \left\{\Delta^{\prime}\left(T_{1}\right)+2 \Delta\left(T_{2}\right), \Delta^{\prime}\left(T_{2}\right)+2 \Delta\left(T_{1}\right)\right\}+1=\Delta^{\prime}\left(T_{1} \square T_{2}\right)+1$.

Let $T_{1}$ and $T_{2}$ be two trees and suppose that one of them has two maximumdegree vertices adjacent to each other. Then the upper and lower bound of $\chi_{s}^{\prime}\left(T_{1} \square T_{2}\right)$ are the same. So the following corollary is immediate.

Corollary 1 Let $T$ be a tree. Then $\chi_{s}^{\prime}\left(T \square P_{n}\right)=2\left(\Delta(T)+\Delta\left(P_{n}\right)\right)=2 \Delta\left(T \square P_{n}\right)$ for every $n>3$, or $n=2$.

In general the upper bound does not hold with equality. An example is the Cartesian product of two stars. Its strong chromatic index is determined in the following theorem. 
Theorem 5 The strong chromatic index of $K_{1, n} \square K_{1, m}$ is $2 n+m+2$, where $n \geq m$.

Proof Let $\left(\{x\},\left\{x_{1}, x_{2}, \ldots, x_{n}\right\}\right)$ and $\left(\{y\},\left\{y_{1}, \ldots, y_{m}\right\}\right)$ be the bipartition of $K_{1, n}$ and $K_{1, m}$ respectively. We first give a strong edge coloring on $K_{1, n} \square K_{1, m}$ with $2 n+m+2$ colors as follows.

$-n+m$ colors on the edges incident with the vertex $x: y$, one dedicated color for each edge.

- $n-m$ colors on the 'long' edges of the $K_{1, n}$-fibers, namely one color for each induced matching of type $\left\{\left(x: y_{j}, x_{i}: y_{j}\right) \mid 1 \leq j \leq m\right\}$, in the range $m+1 \leq i \leq n$.

- $m$ colors on induced matchings which take one edge from each of $n-1 K_{1, m^{-}}$ fibers and one from each of $m-1 K_{1, n}$-fibers, defined as

$$
A_{i}:=\left\{\left(x: y_{i}, x_{j}: y_{i}\right) \mid 1 \leq j \leq n, j \neq i\right\} \cup\left\{\left(x_{i}: y, x_{i}: y_{j}\right) \mid 1 \leq j \leq m, j \neq i\right\}
$$

Hence the vertices of type $x_{i}: y_{j}$ in $A_{i}$ are located in the union of two fibers, namely in $\left(\left\{x_{i}\right\} \times K_{1, m}\right) \cup\left(K_{1, n} \times\left\{y_{i}\right\}\right)$, omitting their intersection vertex $x_{i}: y_{i}$.

- 2 colors for the edges ending in the diagonal vertices $x_{i}: y_{i}$, namely one color for $\left\{\left(x_{i}: y, x_{i}: y_{i}\right) \mid 1 \leq i \leq m\right\}$ and one for $\left\{\left(x: y_{i}, x_{i}: y_{i}\right) \mid 1 \leq i \leq m\right\}$.

It is easily checked that each color class is an induced matching, and their union covers the entire edge set.

Next we show that fewer than $2 n+m+2$ colors are not enough. Note that each of the $n+m$ edges incident with the vertex $x: y$ is adjacent to every other edge. This requires $n+m$ private colors for these edges. Hence it is sufficient to prove that the edges not incident with $x: y$ require more than $n+1$ further colors. Since no color can occur more than once on the edges of any $K_{1, m}$-fiber centered at an $x_{i}: y$, neither on any $K_{1, n}$-fiber centered at an $x: y_{i}$, only the following three types of color classes can occur:

(i) Precisely $m$ edges $\left(x: y_{i}, x_{j_{i}}: y_{i}\right)$ for $i=1,2, \ldots, m$, where $j_{i}$ is arbitrary $\left(1 \leq j_{i} \leq n\right)$; let the number of such color classes be denoted by $a$.

(ii) At most $m-1$ edges incident with vertices of type $x: y_{i}$, and at most $n-1$ edges incident with some of the $x_{i}: y$; let the number of such color classes be denoted by $b$.

(iii) Precisely $n$ edges $\left(x_{i}: y, x_{i}: y_{j_{i}}\right)$ for $i=1,2, \ldots, n$, where $j_{i}$ is arbitrary $\left(1 \leq j_{i} \leq m\right)$; let the number of such color classes be denoted by $c$.

Hence the number of edges involved in such a color partition is distributed as shown in Table 1. (The row correspondng to $b$ gives an upper bound, the other lines are exact.)

The $n$ copies of $K_{1, m}$, as well as the $m$ copies of $K_{1, n}$, contain precisely $m n$ edges. Therefore the following inequalities must hold: 
Table 1 Number of classes, and largest possible number of edges per class

\begin{tabular}{lll}
\hline \# of classes & $K_{1, m}$-edges & $K_{1, n}$-edges \\
\hline$a$ & $m$ & 0 \\
$b$ & $m-1$ & $n-1$ \\
$c$ & 0 & $n$ \\
\hline
\end{tabular}

$$
\begin{gathered}
a m+b(m-1) \geq m n \\
b(n-1)+c n \geq m n
\end{gathered}
$$

Rearrangement yields

$$
a+b \geq n+b / m \quad \text { and } \quad c \geq m-b+b / n
$$

Thus,

$$
a+b+c \geq n+m-b(1-1 / m-1 / n) .
$$

This inequality implies the required lower bound $a+b+c>n+1$, unless

$$
b\left(1-\frac{1}{m}-\frac{1}{n}\right) \geq m-1 .
$$

In this case, however, we have

$$
b \geq \frac{m-1}{1-\frac{1}{m}-\frac{1}{n}}>\frac{m-1}{1-\frac{1}{m}}=m,
$$

from which, using (3), we obtain

$$
a+b \geq n+b / m>n+1 .
$$

Therefore we need at least $2 n+m+2$ colors. Hence $\chi_{s}^{\prime}\left(K_{1, n} \square K_{1, m}\right)=2 n+m+2$.

\section{Cartesian Product of a Tree and a Cycle}

In this section we give lower and upper bounds for the strong chromatic index of the Cartesian product of a tree and a cycle. Also we determine the exact value of that, if the length of the cycle is $4 \ell$ for some $\ell \geq 1$.

Throughout this section $T$ means any tree, and $\Delta$ stands for its maximum degree, as a shorthand for $\Delta(T)$.

Lemma 2 Let $C$ be any cycle of length at least 4 . Then $\chi_{s}^{\prime}(T \square C) \geq 2 \Delta+4$.

Proof Corollary 1 says that the strong chromatic index of the Cartesian product of $T$ and a path longer than two is $2 \Delta+4$. Now $C$ contains a path with 4 vertices. Therefore $\chi_{s}^{\prime}(T \square C) \geq 2 \Delta+4$. 
This inequality holds with equality if the length of the cycle is divisible by 4 . We prove this in the next theorem.

Theorem 6 The strong chromatic index of $T \square C_{4 \ell}$ is $2 \Delta+4$.

Proof The above lemma says that $\chi_{s}^{\prime}\left(T \square C_{4 \ell}\right) \geq 2 \Delta+4$. Now we show that there is a strong edge coloring on $T \square C_{4 \ell}$ that uses $2 \Delta+4$ colors.

Let $a$ be a vertex in $T$ and set $C_{4 \ell}=t_{1} t_{2} \ldots t_{4 \ell} t_{1}$. The proof of Theorem 3 says that there is a strong edge coloring $c$ on $T \square\left(C_{4 \ell} \backslash\left\{\left(t_{4 \ell}, t_{1}\right)\right\}\right)$ with colors $1,2,3, \ldots, 2 \Delta+4$ such that $c$ satisfies the following properties:

(i) All the edges of $T \times V\left(C_{4 \ell}\right)$ use color from $\{1,2,3, \ldots, 2 \Delta\}$.

(ii) All the edges of $V(T) \times C_{4 \ell}$ use color from $\{2 \Delta+1,2 \Delta+2,2 \Delta+3,2 \Delta+4\}$.

(iii) For every $\left(u, u^{\prime}\right) \in E(T), i, j \in[4 \ell-2], \quad$ if $2 \mid(i-j)$, then $c\left(\left(u: t_{i}, u^{\prime}: t_{i}\right)\right)=c\left(\left(u: t_{j}, u^{\prime}: t_{j}\right)\right)$.

(iv) If the distance from $u$ to $u^{\prime}$ is even in $T$, then $c\left(\left(u: t_{i}, u: t_{i+1}\right)\right)=c\left(\left(u^{\prime}: t_{i}, u^{\prime}: t_{i+1}\right)\right)$, for every $i \in[4 \ell-1]$.

Let $b$ be a vertex adjacent to $a$ in $T$. The definition of strong edge coloring ensures that the colors of the edges $\left(a: t_{1}, a: t_{2}\right),\left(a: t_{2}, a: t_{3}\right),\left(b: t_{1}, b: t_{2}\right)$ and $\left(b: t_{2}, b:\right.$ $\left.t_{3}\right)$ are distinct. Again according to (ii) they are $2 \Delta+1,2 \Delta+2,2 \Delta+3,2 \Delta+4$. Without loss of generality we can assume that $c\left(\left(a: t_{1}, a: t_{2}\right)\right)=2 \Delta+1, c((a$ : $\left.\left.t_{2}, a: t_{3}\right)\right)=2 \Delta+2, c\left(\left(b: t_{1}, b: t_{2}\right)\right)=2 \Delta+3$ and $c\left(\left(b: t_{2}, b: t_{3}\right)\right)=2 \Delta+4$.

Now the color of $\left(a: t_{3}, a: t_{4}\right)$ cannot be that of $\left(a: t_{1}, a: t_{2}\right),\left(a: t_{2}, a: t_{3}\right)$ and $\left(b: t_{2}, b: t_{3}\right)$. Hence it is $2 \Delta+3$. Repeating this argument again and again, together with (iv) it leads to the following fact:

Fact $7 c\left(\left(u: t_{i}, u: t_{i+1}\right)\right)=2 \Delta+1+(i-1 \bmod 4)$ if $d_{T}(u, a)$ is even, otherwise it is $24+1+(i-3 \bmod 4)$.

We can extend this coloring to $T \square C_{4 \ell}$ by defining

$$
c\left(\left(u: t_{4 \ell}, u: t_{1}\right)\right):=\left\{\begin{array}{lc}
2 \Delta+2, & \text { if } d_{T}(u, a) \text { is odd } \\
2 \Delta+4, & \text { otherwise }
\end{array}\right.
$$

We claim that this is a strong edge coloring. Since the coloring $c$ satisfies (i), (ii), (iii) and (iv), it is enough to show the following three properties for every vertex $u$ of $T$ and any two vertices $v$ and $v^{\prime}$ adjacent to it.

(a) $c\left(\left(u: t_{1}, v: t_{1}\right)\right) \neq c\left(\left(u: t_{4 \ell}, v^{\prime}: t_{4 \ell}\right)\right)$.

(b) $c\left(\left(u: t_{1}, u: t_{2}\right)\right) \notin\left\{c\left(\left(u: t_{4 \ell}, u: t_{1}\right)\right), c\left(\left(v: t_{4 \ell}, v: t_{1}\right)\right), c\left(\left(u: t_{4 \ell-1}, u: t_{4 \ell}\right)\right)\right\}$

(c)

$$
c\left(\left(u: t_{4 \ell}, u: t_{1}\right)\right) \notin\left\{c\left(\left(u: t_{4 \ell-2}, u: t_{4 \ell-1}\right)\right), c\left(\left(u: t_{2}, u: t_{3}\right)\right), c\left(\left(v: t_{4 \ell}, v: t_{1}\right)\right)\right\}
$$

Now (iii) says that $c\left(\left(u: t_{1}, v: t_{1}\right)\right)=c\left(\left(u: t_{4 \ell-1}, v: t_{4 \ell-1}\right)\right)$. Again $c$ is a strong edge coloring on $T \square\left(C_{4 \ell} \backslash\left\{\left(t_{4 \ell}, t_{1}\right)\right\}\right.$. Hence (a) holds true. Moreover, Fact 7 says that (b) and (c) are valid, too. 
The above theorem determines the strong chromatic index of the Cartesian product of a tree and a cycle whose length is a multiple of 4 . Next we find an upper bound for the Cartesian product of a tree with a cycle of any even length. We state this for the 6-cycle first, and then in Theorem 9 we find a better bound for the remaining even cycles.

Theorem 8 The strong chromatic index of $T \square C_{6}$ is at most $2 \Delta(T)+6$.

Proof Let $a$ be a vertex in $T$, and set $C_{6}=t_{1} t_{2} t_{3} t_{4} t_{5} t_{6} t_{1}$. Consider the strong edge coloring $c$ on $T \square\left(C_{6} \backslash\left\{\left(t_{6}, t_{1}\right)\right\}\right)$ as described in Theorem 6. It follows that the coloring $c$ satisfies the following properties:

(i) All the the edges of $T \times V\left(C_{6}\right)$ use colors from $\{1,2,3, \ldots, 2 \Delta\}$.

(ii) $\quad c\left(\left(u: t_{i}, u^{\prime}: t_{i}\right)\right)=c\left(\left(u: t_{j}, u^{\prime}: t_{j}\right)\right)$, whenever $2 \mid(i-j)$.

We now extend the coloring to obtain the color function $c^{\prime}$ on the edges of $T \square C_{6}$ as follows. Let $c^{\prime}\left(\left(u: t_{i}, v: t_{i}\right)\right)=c\left(\left(u: t_{i}, v: t_{i}\right)\right)$, for all $i$ and $(u, v) \in E(T)$. For every vertex $u$ in $T$, define $c^{\prime}\left(\left(u: t_{i}, u: t_{i+1}\right)\right)$ to be $2 \Delta+1+(i \bmod 3)$ if $d_{T}(u, a)$ is even, else let $2 \Delta+4+(i \bmod 3)$.

Thus for any two adjacent vertices $u$ and $v$ in $T$, we see $c^{\prime}\left(\left(u: t_{i}, u: t_{i+1}\right)\right) \notin$ $\left\{c^{\prime}\left(\left(v: t_{i}, v: t_{i+1}\right)\right), c^{\prime}\left(\left(u: t_{i+1}, u: t_{i+2}\right)\right), c^{\prime}\left(\left(v: t_{i+1}, \quad v: t_{i+2}\right)\right), c^{\prime}\left(\left(u: t_{i+2}\right.\right.\right.$, $\left.\left.\left.u: t_{i+3}\right)\right)\right\}$. Again $c$ is a strong edge coloring and it satisfies (ii). So $c^{\prime}\left(\left(u: t_{i}, v: t_{i}\right)\right) \neq c^{\prime}\left(\left(u: t_{j}, v: t_{j}\right)\right)$, for all $t_{i}$ adjacent to $t_{j}$. Hence $c^{\prime}$ is a strong edge coloring.

This bound is tight. The strong chromatic bound of $C_{6} \square P_{2}$ is 8 . On the other hand there are also graphs whose product does not attend this bound. The strong chromatic index of $C_{6} \square P_{3}$ is 9 .

Theorem 9 Let $\ell>3$. Then $\chi_{s}^{\prime}\left(T \square C_{2 \ell}\right) \leq 2 \Delta(T)+5$.

Proof Let $a$ be a vertex in $T$, and set $C_{2 \ell}=t_{1} t_{2} \ldots t_{2 \ell} t_{1}$. If $\ell$ is even, then Theorem 6 says that the stated bound is true. Now we may assume that $\ell$ is odd. In the proof of Theorem 3 we showed that there is a strong edge coloring $c$ on $T \square\left(C_{2 \ell} \backslash\left\{\left(t_{2 \ell}, t_{1}\right)\right\}\right)$ with colors $1,2,3, \ldots, 2 \Delta+4$ such that $c$ satisfies the following properties:

(i) All the edges from every $T$-fiber use colors from the set $\{1,2,3, \ldots, 2 \Delta\}$.

(ii) All the edges from every $C$-fiber use colors from the set $\{2 \Delta+1,2 \Delta+2,2 \Delta+3,2 \Delta+4\}$.

(iii) $c\left(\left(u: t_{i}, u^{\prime}: t_{i}\right)\right)=c\left(\left(u: t_{i+2}, u^{\prime}: t_{i+2}\right)\right)$, for $\quad$ every $\quad\left(u, u^{\prime}\right) \in E(T)$, $i \leq 2 \ell-2$.

(iv) If the distance from $u$ to $u^{\prime}$ is even in $T$, then $c\left(\left(u: t_{i}, u: t_{i+1}\right)\right)=c\left(\left(u^{\prime}: t_{i}, u^{\prime}: t_{i+1}\right)\right)$, for every $i \in[2 \ell-1]$.

Define the color function $c^{\prime}$ on $T \square V\left(C_{2 \ell}\right)$ as that of $c$. Now (iii) says that $c^{\prime}\left(\left(u: t_{1}, u^{\prime}: t_{1}\right)\right)=c^{\prime}\left(\left(u: t_{2 \ell-1}, u^{\prime}: t_{2 \ell-1}\right)\right)$. Hence $\quad c^{\prime}\left(\left(u: t_{1}, u^{\prime}: t_{1}\right)\right) \neq c^{\prime}((u:$ $\left.\left.t_{2 \ell}, v: t_{2 \ell}\right)\right)$ and $c^{\prime}\left(\left(u: t_{2 \ell}, v: t_{2 \ell}\right)\right) \neq c^{\prime}\left(\left(u: t_{2 \ell-1}, v: t_{2 \ell-1}\right)\right)$, for any vertex $v$ adjacent to $u$ (including $u^{\prime}$ ). Therefore $c^{\prime}$ is a partial coloring on $T \square C_{2 \ell}$ that uses $2 \Delta$ colors. Now we extend this coloring by using 5 new colors. 
Now the only edges which remain to be colored are the edges of $C$-fibers. We partition them into five induced matchings. For every $i \in[2 \ell]$ define the sets $A_{i}:=$ $\left\{\left(u: t_{i}, u: t_{i+1}\right) \mid d_{T}(u, a)\right.$ is even $\}$ and $B_{i}:=\left\{\left(u: t_{i}, u: t_{i+1}\right) \mid d_{T}(u, a)\right.$ is odd $\}$. Clearly every uncolored edge appears exactly in one of the $A_{i}$ or $B_{i}$. Let $\left(u: t_{i}, u\right.$ : $\left.t_{i+1}\right)$ and $\left(v: t_{i}, v: t_{i+1}\right)$ be two edges of $A_{i}$. By the definition of the Cartesian product, $u: t_{i}$ is not adjacent to $v: t_{i+1}$ and $u: t_{i+1}$ is not adjacent to $v: t_{i}$. Again both $d_{T}(u, a)$ and $d_{T}(v, a)$ are even. So $u: t_{i}$ (respectively $u: t_{i+1}$ ) is not adjacent to $v: t_{i}$ (respectively $v: t_{i+1}$ ). Therefore $A_{i}$ is an induced matching, for every $i$. Similarly we can show that for every $i, B_{i}$ is an induced matching. Moreover it satisfies the following properties.

1. For $|i-j|>2 \bmod (2 \ell)$ the sets $A_{i} \cup A_{j}$ and $B_{i} \cup B_{j}$ are induced matchings.

Proof: Let $\left(u: t_{i}, u: t_{i+1}\right) \in A_{i} \quad$ and $\quad\left(v: t_{j}, v: t_{j+1}\right) \in A_{j}$. Since $|i-j|>2 \bmod (2 \ell)$, the definition of the strong edge coloring says that $\{(u$ : $\left.\left.t_{i}, u: t_{i+1}\right),\left(v: t_{j}, v: t_{j+1}\right)\right\}$ is an induced matching. Again both $A_{i}$ and $A_{j}$ are induced matchings. Hence so is $A_{i} \cup A_{j}$. Similarly we can show that $B_{i} \cup B_{j}$ is an induced matching.

2. Let $t_{i}$ be not adjacent to $t_{j}$. Then $A_{i} \cup B_{j}$ and $A_{j} \cup B_{i}$ are induced matchings.

The proof is similar to that of 1 .

We have assumed that $i>3$ and $2 \ell \equiv 2 \bmod 4$. Therefore the above mentioned properties say that the following five sets are induced matchings:

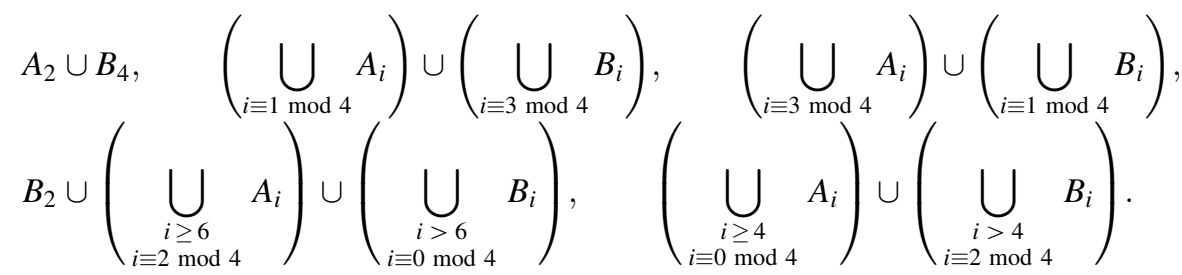

This bound is also tight. The strong chromatic index of $C_{10} \square P_{2}$ is 7 . The above theorem and Theorem 2 say that the strong chromatic index of the Cartesian product of a tree $T$ and an even cycle $C_{2 \ell}$ of length more than 6 is either $2 \Delta(T)+4$ or $2 \Delta(T)+5$. In the next two theorems we prove an upper bound for the strong chromatic index of any tree with cycles of odd length. First we consider cycles of length more than 8 .

Theorem 10 Let $\ell>3$. Then $\chi_{s}^{\prime}\left(T \square C_{2 \ell+1}\right) \leq 2 \Delta(T)+\left\lceil\frac{\Delta(T)}{\ell}\right\rceil+5$.

Proof Let $a$ be a vertex of maximum degree in $T$, and set $C_{2 \ell+1}=t_{1} t_{2} \ldots t_{2 \ell+1} t_{1}$. We view $a$ as the root of $T$. Let $D_{1}, D_{2}, \ldots, D_{\chi_{s}^{\prime}(T)}$ be a partition (some of the $D_{i}$ may be empty) of the edges of $T$ into induced matchings. We are going to define a strong edge coloring $c$ on $T \square C_{2 \ell+1}$. First we give colors to the edges of all the $T$-fibers.

For each $1 \leq i \leq\lfloor\Delta / 2\rfloor$, define the following two $\Delta$-tuples: $c_{2 i+1}:=(\Delta+1, \Delta+$ 
$\left.2, \ldots, \Delta+(i-1)\left\lceil\frac{\Delta}{\ell}\right\rceil, 2 \Delta+1,2 \Delta+2, \ldots, 2 \Delta+\left\lceil\frac{\Delta}{\ell}\right\rceil, i\left\lceil\frac{\Delta}{\ell}\right\rceil+1, i\left\lceil\frac{\Delta}{\ell}\right\rceil+2, \ldots, \Delta\right)$ and $c_{2 i}:=\left(1,2, \ldots,(i-1)\left\lceil\frac{\Delta}{\ell}\right\rceil, \Delta+(i-1)\left\lceil\frac{\Delta}{\ell}\right\rceil+1, \ldots, 2 \Delta\right)$. That is the $j^{\text {th }}$ term of $c_{2 i+1}$,

$$
\left(c_{2 i+1}\right)_{j}=\left\{\begin{array}{cc}
\Delta+j, & 1 \leq j \leq(i-1)\left\lceil\frac{\Delta}{\ell}\right\rceil \\
2 \Delta+j-i+1, & (i-1)\left\lceil\frac{\Delta}{\ell}\right\rceil<j \leq i\left\lceil\frac{\Delta}{\ell}\right\rceil \\
j, & i\left\lceil\frac{\Delta}{\ell}\right\rceil<j \leq \Delta
\end{array}\right.
$$

and the $j^{\text {th }}$ term of $c_{2 i}$,

$$
\left(c_{2 i}\right)_{j}= \begin{cases}j, & 1 \leq j \leq(i-1)\left\lceil\frac{\Delta}{\ell}\right\rceil \\ \Delta+j, & (i-1)\left\lceil\frac{\Delta}{\ell}\right\rceil<j \leq \Delta\end{cases}
$$

Note that $\left(c_{i+1}\right)_{j^{\prime}} \neq\left(c_{i}\right)_{j}$ and $\left(c_{i}\right)_{j} \neq\left(c_{i}\right)_{j^{\prime}}$, for all $i, j, j^{\prime}$.

Now for any vertex $u^{\prime}$ and its parent $u$ in $T$, define

$$
c\left(\left(u: t_{i}, u^{\prime}: t_{i}\right)\right):= \begin{cases}\left(c_{i}\right)_{j}, & \text { if } d_{T}(a, u) \text { is even and }\left(u, u^{\prime}\right) \in D_{j} \\ \left(c_{i+1}\right)_{j}, & \text { if } d_{T}(a, u) \text { is odd and }\left(u, u^{\prime}\right) \in D_{j}\end{cases}
$$

We claim that each color class is an induced matching in $T \square C_{2 \ell+1}$.

Let $\left(u: t_{i}, u^{\prime}: t_{i}\right)$ and $\left(u: t_{i}, u^{\prime \prime}: t_{i}\right)$ be two adjacent edges. Then, since every $D_{i}$ is an induced matching, both $\left(u, u^{\prime}\right)$ and $\left(u, u^{\prime \prime}\right)$ cannot be in the same $D_{i}$. Hence $c\left(\left(u: t_{i}, u^{\prime}: t_{i}\right)\right) \neq c\left(\left(u: t_{i}, u^{\prime \prime}: t_{i}\right)\right)$. Now let $v: t_{k}$ be adjacent to $u: t_{i}$. The definition of the Cartesian product says that either $i=k$ or $u=v$. If $i=k$, then $c\left(\left(u: t_{i}, u^{\prime}: t_{i}\right)\right) \neq c\left(\left(v: t_{i}, v^{\prime}: t_{i}\right)\right)$, for any $v^{\prime}$ (using the properties of the sets $c_{j}$, and that the $D_{i}$ are induced matchings). If $i \neq k$, then $u=v$ and $t_{i}$ is adjacent to $t_{j}$. Without loss of generality, let $k=i+1 \bmod (2 \ell+1)$. We know that $\left(c_{i}\right)_{j} \neq\left(c_{i+1}\right)_{j^{\prime}}$, for all $i, j, j^{\prime}$. So $c\left(\left(u: t_{i}, u^{\prime}: t_{i}\right)\right) \neq c\left(\left(v: t_{i}, v^{\prime}: t_{i}\right)\right)$. Therefore every color class is an induced matching.

The remaining (uncolored) edges are the edges of all the $C$-fibers. Therefore it is sufficient to partition them into five induced matchings. As in the proof of the earlier theorem, we partition them into the sets $A_{i}:=\left\{\left(u: t_{i}, u: t_{i+1}\right) \mid d_{T}(u, a)\right.$ is even $\}$ and $B_{i}:=\left\{\left(u: t_{i}, u: t_{i+1}\right) \mid d_{T}(u, a)\right.$ is odd $\}, i \in[2 \ell+1]$. It is easy to see that the following properties hold.

(i) For every $i, A_{i}$ and $B_{i}$ are induced matchings.

(ii) Let $|i-j|>2 \bmod (2 \ell+1)$. Then $A_{i} \cup A_{j}$ and $B_{i} \cup B_{j}$ are induced matchings.

(iii) Let $t_{i}$ is not adjacent to $j$. Then $A_{i} \cup B_{j}$ and $A_{j} \cup B_{i}$ are induced matchings.

Now $B_{1} \cup A_{5} \cup B_{7}$ is an induced matching because $2 \ell+1 \equiv 1$ or $3 \bmod 4$. Therefore it is enough to partition the rest of the edges into four induced matchings. If $2 \ell+1 \equiv 1 \bmod 4$, then the sets 


$$
\begin{aligned}
& A_{1} \cup B_{3} \cup\left(\bigcup_{\substack{i>6 \\
i \equiv 0 \bmod 4}} A_{i}\right) \cup\left(\bigcup_{\substack{i \geq 6 \\
i \equiv 2 \bmod 4)}} B_{i}\right), \quad\left(\bigcup_{i \equiv 2 \bmod 4} A_{i}\right) \cup\left(\bigcup_{\substack{i \geq 7 \\
i \equiv 3 \bmod 4}} A_{i} B_{i}\right), \\
& B_{2} \cup A_{4} \cup\left(\bigcup_{\substack{i>7 \\
i \equiv 1 \bmod 4}} B_{i}\right), \\
& A_{3} \cup B_{5} \cup\left(\bigcup_{\substack{i \geq 9 \\
i \equiv 1 \bmod 4}} A_{i}\right) \cup\left(\bigcup_{i>9} B_{i}\right)
\end{aligned}
$$

partition $V(T) \times C_{2 \ell+1}$ into induced matchings. Otherwise $2 \ell+1 \equiv 3 \bmod 4$, and then the sets

$$
\begin{aligned}
& B_{2} \cup A_{4} \cup\left(\bigcup_{\substack{i>6 \\
i \equiv 0 \bmod 4}} A_{i}\right) \cup\left(\bigcup_{\substack{i \geq 6 \\
i \equiv 2 \bmod 4)}} B_{i}\right), \quad\left(\bigcup_{i \equiv 2 \bmod 4} A_{i}\right) \cup\left(\bigcup_{\substack{i \geq 7 \\
i \equiv 3 \bmod 4}} A_{i} B_{i}\right), \\
& A_{2} \cup B_{4} \cup\left(\bigcup_{\substack{i>7 \\
i \equiv 1 \bmod 4}} B_{i}\right), \\
& A_{3} \cup B_{5} \cup\left(\bigcup_{\substack{i \geq 9 \\
i \equiv 1 \bmod 4}} A_{i}\right) \cup\left(\bigcup_{i>9}^{j} B_{i}\right),
\end{aligned}
$$

form a required partition.

Therefore the strong chromatic index of $T \square C_{2 \ell+1}$ is at most $2 \Delta+\left\lceil\frac{\Delta}{\ell}\right\rceil+5$, for $\ell>3$.

Above we obtained bounds for the strong chromatic index of a tree with a cycle of length different from 3,5 and 7. Now we find a slightly larger upper bound for the same when a cycle is of length 3,5 and 7.

Theorem 11 Let $\ell \leq 3$. Then $\chi_{s}^{\prime}\left(T \square C_{2 \ell+1}\right) \leq 2 \Delta(T)+\left\lceil\frac{\Delta(T)}{\ell}\right\rceil+6$.

Proof Let $a$ be an arbitrary vertex of $T$. We can assign the colors $1,2,3, \ldots$, $2 \Delta+\left\lceil\frac{\Delta}{\ell}\right\rceil$ to the edges of $T \times V\left(C_{2 \ell+1}\right)$ in the same way as in the proof of the previous theorem. The only edges which remain to be colored are the edges of $V(T) \times C_{2 \ell+1}$.

Define $A_{i}$ and $B_{i}$ as in the preceding proof, for $i \leq 2 \ell+1$. We have already shown above that the sets $A_{i}$ and $B_{i}$ are induced matchings. Hence $\chi_{s}^{\prime}\left(T \square C_{3}\right) \leq 3 \Delta+6$. Now further properties of these $A_{i}$ and $B_{i}$ (as mentioned above) ensure that the following properties are true.

If $\ell=2$, then $A_{1} \cup B_{3}, B_{1} \cup A_{3}, A_{2} \cup B_{4}, B_{2} \cup A_{4}, A_{5}$, and $B_{5}$ are induced 
matchings; and if $\ell=3$, then $A_{1} \cup B_{3} \cup A_{5}, \quad B_{1} \cup A_{3} \cup B_{5}, \quad A_{2} \cup B_{4} \cup A_{6}$, $B_{2} \cup A_{4} \cup B_{6}, \quad A_{7}$, and $B_{7}$ are induced matchings. Therefore $\chi_{s}^{\prime}\left(T \square C_{2 \ell+1}\right) \leq 2 \Delta+\left\lceil\frac{\Delta}{\ell}\right\rceil+6$, for $\ell \leq 3$.

Finally, we give a better lower bound for the same when the cycle is of odd length. For that, first we define jellyfish graphs. Let $H$ be a graph obtained from $C_{k}$ by adding $p_{v}$ new pendant vertices adjacent to $v$, for each vertex $v$ in $C_{k}$. Then $H$ is called a $C_{k}$-jellyfish. On such graphs, Chang et al. [11] proved a general lower bound (their Theorem 13), which implies the following formula.

Theorem 12 [11] If $G$ is a $C_{k}$-jellyfish of $m$ edges, such that $k$ is odd and all vertices of $C_{k}$ have the same degree in $G$, then $\chi_{s}^{\prime}(G) \geq\left\lceil\frac{m}{[k / 2\rceil}\right\rceil$.

By using this theorem, we can derive an improved lower bound for the strong chromatic index of the product of a tree with an odd cycle.

Corollary 2 If $T$ is a tree, then $\chi_{s}^{\prime}\left(T \square C_{2 \ell+1}\right) \geq\left\lceil\frac{(2 \ell+1)(\Delta(T)+1)}{\ell}\right\rceil=2 \Delta+\left\lceil\frac{\Delta(T)+1}{\ell}\right\rceil+2$.

Proof Let $u$ be a vertex of maximum degree in $T$, and set $C_{2 \ell+1}=t_{1} t_{2} \ldots t_{2 \ell+1} t_{1}$. Now $T \square C_{2 \ell+1}$ contains $G[N(u) \cup\{u\}] \square C_{2 \ell+1}$, which contains a $(\Delta+2)$-regular $C_{2 \ell+1}$-jellyfish. Therefore $\chi_{s}^{\prime}\left(T \square C_{2 \ell+1}\right) \geq\left\lceil\frac{(2 \ell+1)(\Delta+1)}{\ell}\right\rceil$.

Remark 1 Note that the difference of the lower and the upper bound for the strong chromatic index of a tree with an odd cycle is at most 4 . In fact, if the odd cycle is of length at least 9 , then the difference is at most 3 .

\section{Conclusion}

Table 2 summarizes the results which involve cycles.

Theorem 11 and Corollary 2 give upper and lower bounds for the strong chromatic index of product of any tree with odd cycles. In particular for $C_{3}$ the strong chromatic index of both $K_{2} \square C_{3}$ and $P_{3} \square C_{3}$ is 9 , showing that the upper bound $2 \Delta+\left\lceil\frac{\Delta}{\ell}\right\rceil+6$ and also the lower bound $2 \Delta+\left\lceil\frac{\Delta+1}{\ell}\right\rceil+2$ is tight in case of $\ell=1$ for at least one graph (with $\Delta=1$ and $\Delta=2$, respectively). Therefore the bounds for the strong chromatic index of product of a tree with $C_{3}$ cannot be

Table 2 Upper and lower bounds on $\chi_{s}^{\prime}\left(T \square C_{k}\right)$ for all trees $T$, where $\Delta:=\Delta(T)$

\begin{tabular}{lllll}
\hline cycle length $k=$ & upper bound & theorem & lower bound & result \\
\hline $4 \ell$ & $2 \Delta+4$ & 6 & $2 \Delta+4$ & Lemma 2 \\
6 & $2 \Delta+6$ & 8 & & \\
$4 \ell+2 \geq 10$ & $2 \Delta+5$ & 9 & & \\
$2 \ell+1 \leq 7$ & $2 \Delta+\left\lceil\frac{\Delta}{\ell}\right\rceil+6$ & 11 & $2 \Delta+\left\lceil\frac{\Delta+1}{\ell}\right\rceil+2$ & Corollary 2 \\
$2 \ell+1 \geq 9$ & $2 \Delta+\left\lceil\frac{\Delta}{\ell}\right\rceil+5$ & 10 & & \\
\hline
\end{tabular}


improved in general. For other odd cycles these bounds might be improved. The tightness of bounds for the strong chromatic index of product of trees with even cycles was discussed in the previous section.

Note Added in Proof. Recently the authors proved the generalization of Theorem 3 for the Cartesian product of any number of trees.

Funding Open access funding provided by ELKH Alfréd Rényi Institute of Mathematics. The fourth author thanks for the support given in part by the Széchenyi 2020 programme under the project No. EFOP-3.6.1-16-2016-00015, and by the National Research, Development and Innovation Office - NKFIH under the grant SNN 129364.

Availability of data and material Not applicable.

Code availability Not applicable.

\section{Declarations}

Conflict of interest The authors declare that they have no conflict of interest.

Open Access This article is licensed under a Creative Commons Attribution 4.0 International License, which permits use, sharing, adaptation, distribution and reproduction in any medium or format, as long as you give appropriate credit to the original author(s) and the source, provide a link to the Creative Commons licence, and indicate if changes were made. The images or other third party material in this article are included in the article's Creative Commons licence, unless indicated otherwise in a credit line to the material. If material is not included in the article's Creative Commons licence and your intended use is not permitted by statutory regulation or exceeds the permitted use, you will need to obtain permission directly from the copyright holder. To view a copy of this licence, visit http:// creativecommons.org/licenses/by/4.0/.

\section{References}

1. Akhtar, R., Boggess, M., Jackson-Henderson, T., Karpman, R., Jiménez, I., Kinzel, A., Pritikin, D.: On the unitary Cayley graph of a finite ring. Electron. J. Combin. 16, \#R117 (2009)

2. Alon, Noga: An extremal problem for sets with applications to graph theory. J. Combin. Theory Ser A 40(1), 82-89 (1985)

3. Andersen, Lars Døvling: The strong chromatic index of a cubic graph is at most 10. Discret. Math. 108(1-3), 231-252 (1992)

4. Bensmail, Julien, Bonamy, Marthe, Hocquard, Hervé: Strong edge coloring sparse graphs. Electron. Notes Discret. Math. 49, 773-778 (2015)

5. Bermond, J.C., Bond, J., Paoli, M., Peyrat, C.: Graphs and interconnection networks: diameter and vulnerability. In: Lloyd, E.K. (ed.) Surveys in Combinatorics, volume 82 of London Mathematical Society Lecture Notes, pp. 1-30. Cambridge University Press (1983)

6. Berrizbeitia, P., Giudici, R.E.: On cycles in the sequence of unitary cayley graphs. Discret. Math. 282, 1-3 (2004)

7. Borozan, Valentin, Montero, Leandro, Narayanan, Narayanan: Further results on strong edgecolourings in outerplanar graphs. Australas. J. Combin. 62(1), 35-44 (2015)

8. Brualdi, R.A., Massey, J.J.Q.: Incidence and strong edge colorings of graphs. Discret. Math. 122(1-3), 51-58 (1993)

9. Bruhn, H., Joos, F.: A stronger bound for the strong chromatic index. Electron. Notes Discret. Math. 49, 277-284 (2015)

10. Chalermsook, P., Laekhanukit, B., Nanongkai, D.: Coloring graph powers: Graph product bounds and hardness of approximation. In: Alberto, P., Alfredo, V. (eds.) LATIN 2014: Theoretical Informatics. Lecture Notes in Computer Science, vol. 8392, pp. 409-420. Springer, Berlin, Heidelberg (2014) 
11. Chang, G.J., Chen, S.-H., Hsu, C.-Y., Hung, C.-M., Lai, H.-L.: Strong edge-coloring for jellyfish graphs. Discret. Math. 338(12), 2348-2355 (2015)

12. Chung, F.R.K., Gyárfás, A., Trotter, W.T., Tuza, Zs.: The maximum number of edges in $2 K_{2}$-free graphs of bounded degree. Discret. Math. 81, 129-135 (1990)

13. Cranston, D.W.: Strong edge-coloring of graphs with maximum degree 4 using 22 colors. Discret. Math. 306(21), 2772-2778 (2006)

14. Dejter, J., Giudici, R.E.: On unitary Cayley graphs. J. Combin. Math. Combin. Comput. 18, 121-124 (1995)

15. Erdős, P.: Problems and results in combinatorial analysis and graph theory. Discret. Math. 72, 81-92 (1988)

16. Faudree, R.J., Gyárfás, A., Schelp, R.H., Tuza, Z.: Induced matchings in bipartite graphs. Discret. Math. 78(1-2), 83-87 (1989)

17. Faudree, R.J., Gyárfás, A., Schelp, R.H., Tuza, Zs.: The strong chromatic index of graphs. Ars Combin. 29B, 205-211 (1990)

18. Fouquet, J.L., Jolivet, J.L.: Strong edge-coloring of graphs and applications to multi- $k$-gons. Ars Combin. 16A, 141-150 (1983)

19. Horák, P.: The strong chromatic index of graphs with maximum degree four. In: Rainer, B., Klaus, W. (eds.) Contemporary Methods in Graph Theory, pp. 399-403. Wissenschaftsverlag, BI (1990)

20. Horák, P., Qing, H., Trotter, W.T.: Induced matchings in cubic graphs. J Graph Theory 17(2), 151-160 (1993)

21. Hu, Z., Lih, K.-W., Liu, D.D.-F.: Upper bounds for the strong chromatic index of Halin graphs. Discuss. Math. Graph Theory 38(1), 5-26 (2018)

22. Huang, M., Santana, M., Yu, G.: Strong chromatic index of graphs with maximum degree four. Eectron. J. Combin. 25(3), \#P3.31 (2018)

23. Huang, M., Gexin, Y., Zhou, X.: The strong chromatic index of $(3, \delta)$-bipartite graphs. Discret. Math. 340(5), 1143-1149 (2017)

24. Hudák, D., Lužar, B., Soták, R., Škrekovski, R.: Strong edge-coloring of planar graphs. Discret. Math. 324, 41-49 (2014)

25. Hurley, E., de Joannis de Verclos, R., Kang, R.J.: An improved procedure for colouring graphs of bounded local density. In Proceedings of the 2021 ACM-SIAM Symposium on Discrete Algorithms (SODA), pp. 135-148. SIAM (2021)

26. Imrich, W., Klavžar, S., Rall, D.F.: Topics in Graph Theory: Graphs and Their Cartesian Product. AK Peters/CRC Press (2008)

27. Klotz, W., Sander, T.: Some properties of unitary Cayley graphs. Electron. J. Combin. 14, \#R45 (2007)

28. Lai, H-H., Lih, K-W., Tsai, P-Y.: The strong chromatic index of Halin graphs. Discret. Math. 312(9), $1536-1541$ (2012)

29. Ko-Wei L., Daphne D-F.L: The strong chromatic index of cubic Halin graphs. Appl. Math. Lett. 25(5), 898-901 (2012)

30. Lv, J.-B., Li, X., Gexin, Y.: On strong edge-coloring of graphs with maximum degree 4. Discret. Appl. Math. 235, 142-153 (2018)

31. Mahdian, M.: On the computational complexity of strong edge coloring. Discret. Appl. Math. 118(3), 239-248 (2002)

32. Molloy, M., Reed, B.: A bound on the strong chromatic index of a graph. J. Combin. Theory Ser B 69(2), 103-109 (1997)

33. Muthu, R., Narayanan, N., Subramanian, C.R.: On $k$-intersection edge colourings. Discuss. Math. Graph Theory 29(2), 411-418 (2009)

34. Nakprasit, K.: A note on the strong chromatic index of bipartite graphs. Discret. Math. 308(16), 3726-3728 (2008)

35. Togni, O: Strong chromatic index of products of graphs. Discret. Math. Theor. Comput. Sci. 9(1), 47-56 (2007)

Publisher's Note Springer Nature remains neutral with regard to jurisdictional claims in published maps and institutional affiliations. 